\title{
SUPER-RESOLUTION FROM HIGHLY UNDERSAMPLED IMAGES
}

\author{
P. Vandewalle, L. Sbaiz, M. Vetterli, S. Süsstrunk \\ Ecole Polytechnique Fédérale de Lausanne (EPFL) \\ School of Computer and Communication Sciences \\ CH-1015 Lausanne, Switzerland
}

\begin{abstract}
Aliasing artifacts in images are visually very disturbing. Therefore, most imaging devices apply a low-pass filter before sampling. This removes all aliasing from the image, but it also creates a blurred image. Actually, all the image information above half the sampling frequency is removed. In this paper, we present a new method for the reconstruction of a high resolution image from a set of highly undersampled and thus aliased images. We use the information in the entire frequency spectrum, including the aliased part, to create a sharp, high resolution image. The unknown relative shifts between the images are computed using a subspace projection approach. We show that the projection can be decomposed into multiple projections onto smaller subspaces. This allows for a considerable reduction of the overall computational complexity of the algorithm. A high resolution image can then be reconstructed from the registered low resolution images. Simulation results show the validity of our algorithm.
\end{abstract}

\section{INTRODUCTION}

Known for more than fifty years, the Shannon sampling theorem states that a signal can be perfectly reconstructed from its samples if the sampling frequency is larger than twice the maximum signal frequency. If the signal is sampled at a lower frequency, the sampled signal is aliased, and the original signal can generally not be reconstructed. However, all the frequency content is still present in the sampled signal. It would therefore be interesting to use all this frequency information to reconstruct the signal. In this paper, we will show a perfect reconstruction algorithm using multiple sets of arbitrarily undersampled signals. The different sets of samples have unknown, real-valued relative offsets.

The work presented in this paper was supported by the National Competence Center in Research on Mobile Information and Communication Systems (NCCR-MICS, http://www.mics.org), a center supported by the Swiss National Science Foundation under grant number 5005-67322.

Martin Vetterli is also with the Department of Electrical Engineering and Computer Science, UC Berkeley, Berkeley CA94720.
A similar problem for discrete-valued offsets is described by Marziliano and Vetterli [1]. They compute the relative offsets using a combinatorial method. Another method for solving this problem with bandlimited signals is described in [2]. The relative offsets are computed using a subspace algorithm in frequency domain.

In imaging, such algorithms are generally used to solve the super-resolution problem. A number of low resolution images, either registered or not, is combined to reconstruct a higher resolution image. The super-resolution idea was introduced in 1984 by Tsai and Huang [3]. A good overview of existing algorithms is given by Borman and Stevenson [4] and by Park et al. [5].

In this paper, we will first formulate the problem mathematically (Section 2) and solve it for one-dimensional signals (Section 3). It is then extended to images in Section 5. Results are shown in Section 6 and discussed in Section 7.

\section{PROBLEM STATEMENT FOR 1D SIGNALS}

Take a periodic, bandlimited, continuous-time signal $x(t)$ with period 1 and Fourier coefficients $X[l](-L \leq l \leq L)$. We want to reconstruct $x(t)$ from $M$ sets of samples $y_{m}[n]$ $(0 \leq m<M, 0 \leq n<N)$ that are all undersampled. The sampling frequency $N$ for obtaining the different $y_{m}[n]$ does not satisfy the Nyquist criterium: $N \leq 2 L$. The sets of samples can thus be written as

$$
y_{m}[n]=x\left(\frac{n+t_{m}}{N}\right) \text {, }
$$

with $t_{m}$ the offset of the $m$-th set of samples relative to the first one $\left(t_{0}=0\right)$. In our setup, we assume that next to the $M$ sets of samples $y_{m}[n]$, the sampling frequency $N$ and the maximum signal frequency $L$ are known. We want to compute the original Fourier coefficients $X[l]$, and for this, the offsets $\left\{t_{m}\right\}_{m=0 . . M-1}$ need to be determined first. 


\section{SOLUTION FOR 1D SIGNALS}

The reference set of samples $y_{0}[n]$ can be written as a function of the unknown Fourier coefficients as

$$
y_{0}[n]=\sum_{l=-L}^{L} X[l] e^{\frac{j 2 \pi l n}{N}} .
$$

As $N \leq 2 L$, different spectrum components overlap, and $x(t)$ cannot be perfectly reconstructed from $y_{0}[n]$. Therefore, $M$ sets of samples are considered, with unknown offsets $t_{m}$. The $m$-th set of samples can be written as

$$
y_{m}[n]=\sum_{l=-L}^{L} X[l] e^{\frac{j 2 \pi l\left(t_{m}+n\right)}{N}}=\sum_{l=-L}^{L} X[l] W_{N}^{l t_{m}+l n}
$$

with $W_{N}=e^{j 2 \pi / N}$. In matrix notation, we obtain

$$
\begin{aligned}
& {\left[\begin{array}{c}
y_{m}[0] \\
y_{m}[1] \\
\vdots \\
y_{m}[N-1]
\end{array}\right]=\left[\begin{array}{cccc}
W_{N}^{-L t_{m}} & \cdots & \cdots & W_{N}^{L t_{m}} \\
W_{N}^{-L t_{m}-L} & \cdots & \cdots & W_{N}^{L t_{m}+L} \\
\vdots & \vdots & \vdots \\
W_{N}^{-L t_{m}-L(N-1)} & \cdots & \cdots & W_{N}^{L t_{m}+L(N-1)}
\end{array}\right]\left[\begin{array}{c}
X[-L] \\
\vdots \\
X[0] \\
X[L]
\end{array}\right]} \\
& \text { ^ } \\
& \boldsymbol{y}_{m}=\mathbf{W}_{m} \mathbf{X} \text {. }
\end{aligned}
$$

The different sets of samples can be combined in a large matrix-vector product as

$$
\begin{aligned}
{\left[\begin{array}{c}
\boldsymbol{y}_{0} \\
\boldsymbol{y}_{1} \\
\vdots \\
\boldsymbol{y}_{M-1}
\end{array}\right] } & =\left[\begin{array}{c}
\mathbf{W}_{0} \\
\mathbf{W}_{1} \\
\vdots \\
\mathbf{W}_{M-1}
\end{array}\right] \mathbf{X} \\
& \Uparrow \\
\boldsymbol{y} & =\mathbf{W X},
\end{aligned}
$$

with $\boldsymbol{y}$ a $M N \times 1$ vector, $\mathbf{W}$ a $M N \times(2 L+1)$ matrix, and $\mathbf{X}$ a $(2 L+1) \times 1$ vector. From (5), we can see that the combined sample vector $\boldsymbol{y}$ belongs to the $2 L+1$-dimensional space spanned by the columns of $\mathbf{W}$. The matrix $\mathbf{W}$ depends on the unknown offsets $\left\{t_{m}\right\}_{m=0 . . M-1}$. Therefore, we can find these offsets as the values for which the sample vector $y$ remains unchanged under projection onto the space spanned by $\mathbf{W}$. In other words, we can compute $\left\{t_{m}\right\}_{m=0 . . M-1}$ as

$$
\begin{gathered}
\min _{\left\{t_{m}\right\}}\|\boldsymbol{y}-\hat{\boldsymbol{y}}\|_{2}^{2} \\
\text { with } \hat{\boldsymbol{y}}=P_{\mathbf{W}} \boldsymbol{y}=\mathbf{W}\left(\mathbf{W}^{T} \mathbf{W}\right)^{-1} \mathbf{W}^{T} \boldsymbol{y} .
\end{gathered}
$$

The matrix $P_{\mathbf{W}}$ performs a projection onto the space spanned by the columns of $\mathbf{W}$. Once the offsets $\left\{t_{m}\right\}_{m=0 . . M-1}$ are known, the original signal $x(t)$ can be reconstructed. In (5), $\mathbf{W}$ is now known, and there are $M N$ linear equations in $2 L+1$ unknown Fourier coefficients $X[l]$. In order to have a unique solution for this set of equations, we require that the number of sample sets $M$ has to satisfy

$$
M>(2 L+1) / N \text {. }
$$

The solution of these equations can be computed using a least squares method to make it more robust to noise.

\section{INTERPRETATION}

The computation of the relative offsets forms the central part of our reconstruction algorithm. An example of $\|\boldsymbol{y}-\hat{\boldsymbol{y}}\|_{2}^{2}$, the function to minimize, is shown in Figure 1 for two and three sets of samples ( $M=2$ and $M=3$ ). Next to the global minimum, this function also has a large number of local minima. It is therefore important to understand its structure.

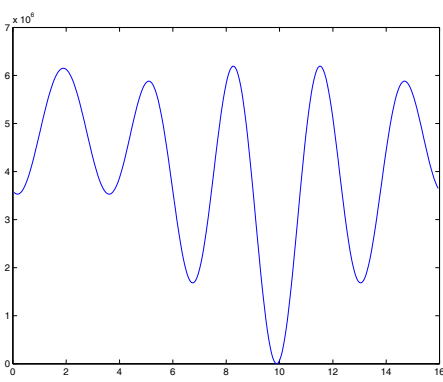

(a)

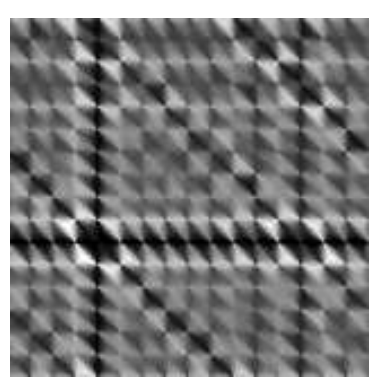

(b)
Fig. 1. Examples of $\|\boldsymbol{y}-\hat{\boldsymbol{y}}\|_{2}^{2}$ for one-dimensional signals. Next to the global minimum, many local minima are visible. (a) Two sets of samples. $\|\boldsymbol{y}-\hat{\boldsymbol{y}}\|_{2}^{2}$ for different values of $t_{1}$. (b) Three sets of samples. $\|\boldsymbol{y}-\hat{\boldsymbol{y}}\|_{2}^{2}$ for different values of $t_{1}$ and $t_{2}$. Brighter pixels represent higher values.

Let us first analyze the simplest case, where signals are undersampled by a factor less than two. This means that there are at most two overlapping spectral components at each frequency (see Figure 2). The undersampling factor has to be strictly smaller than two, because we need some extra information to be able to compute the offsets $\left\{t_{m}\right\}_{m=0 . . M-1}$. In this case, two sets of samples are sufficient for perfect reconstruction $(M=2)$, as it can be seen from (7).

Each column $\mathbf{w}_{l}$ of $\mathbf{W}$ can be written as

$\left[1 W_{N}^{l} W_{N}^{2 l} \cdots W_{N}^{(N-1) l} W_{N}^{l t_{1}} W_{N}^{l t_{1}+l} \cdots W_{N}^{l t_{1}+(N-1) l}\right]^{T}$,

with $-L \leq l \leq L$. If we denote by $A_{l}$ the vector

$$
A_{l}=\left[\begin{array}{lllll}
1 & W_{N}^{l} & W_{N}^{2 l} & \cdots & W_{N}^{(N-1) l}
\end{array}\right]^{T},
$$




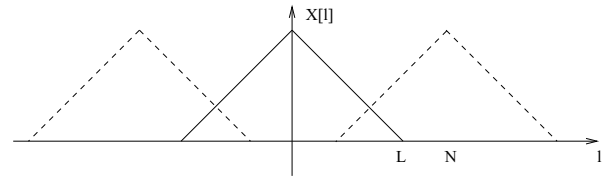

Fig. 2. Aliasing with a factor less than two. The original spectrum (-) and two aliased copies (- -) are displayed

we can rewrite $(8)$ as

$$
\mathbf{w}_{l}=\left[\begin{array}{ll}
A_{l} & W_{N}^{l t_{1}} A_{l}
\end{array}\right]^{T} .
$$

It describes a trajectory in a two-dimensional subspace of $\mathcal{C}^{2 N}$, as a function of the unknown offset $t_{1}$, namely the space

$$
\mathcal{S}_{l}=\operatorname{span}\left(\left[\begin{array}{l}
A_{l} \\
0
\end{array}\right],\left[\begin{array}{l}
0 \\
A_{l}
\end{array}\right]\right) .
$$

If $-L \leq l \pm N \leq L$ (i.e. two components overlap), column $\mathbf{w}_{l}$ and column $\mathbf{w}_{l \pm N}$ describe the same trajectory. This can be seen from the fact that since $W_{N}^{N}=1$, in (9) $A_{l}=$ $A_{l \pm N}$ and therefore in (10), $\mathbf{w}_{l}$ and $\mathbf{w}_{l \pm N}$ only differ by their coefficient $W_{N}^{t_{1}}$. In this case $\mathcal{S}_{l}=\mathcal{S}_{l \pm N}$.

If $t_{1} \neq 0$, these two vectors $\mathbf{w}_{l}$ and $\mathbf{w}_{l \pm N}$ form a basis for the two-dimensional subspace $\mathcal{S}_{l}$. Each of these $N$ two-dimensional subspaces $\mathcal{S}_{l}$ is orthogonal to all other subspaces $\mathcal{S}_{i, i \neq l}$. This is obvious from (11) and the fact that $A_{l}$ and $A_{i}$ are (orthogonal) Fourier basis vectors. The projection of the sample vector $\boldsymbol{y}$ onto $\mathbf{W}$ can therefore be decomposed into separate projections onto each of these $N$ known two-dimensional subspaces. We can rewrite (6) as

$\|\boldsymbol{y}-\hat{\boldsymbol{y}}\|_{2}^{2}=\left\|\boldsymbol{y}_{0}-\hat{\boldsymbol{y}}_{0}\right\|_{2}^{2}+\left\|\boldsymbol{y}_{1}-\hat{\boldsymbol{y}}_{1}\right\|_{2}^{2}+\cdots+\left\|\boldsymbol{y}_{N-1}-\hat{\boldsymbol{y}}_{N-1}\right\|_{2}^{2}$

If at frequency $i$, there are two overlapping frequency components, the subspace $\mathcal{S}_{i}$ is spanned by the two vectors $\mathbf{w}_{i}$ and $\mathbf{w}_{i \pm N}$, and therefore

$$
\left\|\boldsymbol{y}_{i}-\hat{\boldsymbol{y}}_{i}\right\|_{2}^{2}=0
$$

Thus, (6) can be rewritten using only the subspaces $\mathcal{S}_{i}$ where there is only one frequency component:

$$
\|\boldsymbol{y}-\hat{\boldsymbol{y}}\|_{2}^{2}=\sum_{i \in \mathcal{L}}\left\|\boldsymbol{y}_{i}-\hat{\boldsymbol{y}}_{i}\right\|_{2}^{2}
$$

with $\mathcal{L}$ the set of frequencies that do not overlap. An example is shown in Figure 3. The global minimum is found as the value of $t_{1}$ for which the (periodic) minima of the different components coincide.

More generally, when at most $S$ components overlap and $M$ sets of samples are used, the columns describe trajectories in $M$-dimensional subspaces of $\mathcal{C}^{M N}$. The offsets $\left\{t_{m}\right\}_{m=0 . . M-1}$ can therefore be searched separately in the

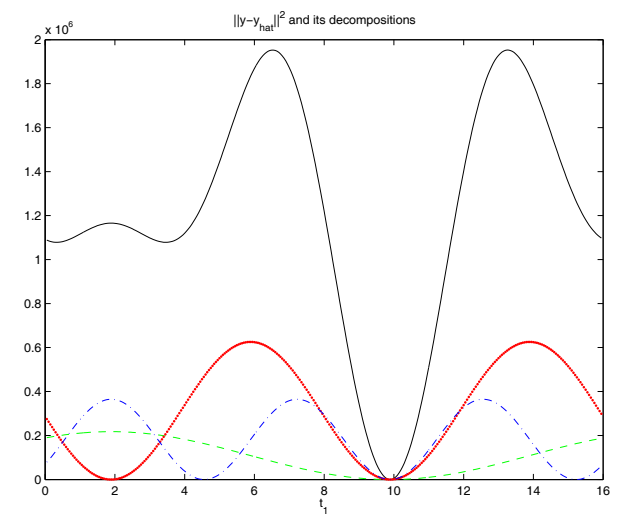

Fig. 3. Decomposition of $\|\boldsymbol{y}-\hat{\boldsymbol{y}}\|_{2}^{2}$ (solid line) in its components from different two-dimensional subspaces.

different subspaces having less than $M$ overlapping components. In each $M$-dimensional subspace, we first project $\boldsymbol{y}$ onto the $M$-dimensional subspace described by the trajectory of the overlapping columns of $\mathbf{W}$. Next, we search for the particular set of values $\left\{t_{m}\right\}_{m=0 . . M-1}$, such that the projection belongs to the $S$-dimensional subspace corresponding to these offset values. The global solution is found as the common solution for all the components in the different $M$-dimensional subspaces.

\section{EXTENSION TO 2D SIGNALS}

The above method can be directly applied to superresolution imaging. In this simulation, we assume there are only global horizontal and vertical shifts between the different images. The images are undersampled by a factor $S$ in both dimensions, and therefore there are at most $S^{2}$ overlapping spectrum components. The horizontal and vertical offsets can be computed independently, considering first the lines and then the columns of the image. To compute the Fourier coefficients of the high resolution image when the offsets are known, we need to have at least $M=\left\lceil((2 L+1) / N)^{2}\right\rceil$ input images.

\section{RESULTS}

Our algorithm was tested on some real images in a simulation. Four low resolution, (circularly) shifted and aliased images are created from an original high resolution image. In our simulation, we undersampled the images by almost two in both dimensions. The undersampling has to be slightly smaller than two, because the offsets also have to be estimated. If the images are undersampled by exactly two, the set of equations is underdetermined due to the unknown offsets. In our simulation, the relative offsets were computed exactly and the high resolution image was recon- 
structed from the low resolution images up to working precision (Figure 4, RMSE $=7 \cdot 10^{-12}$ ).

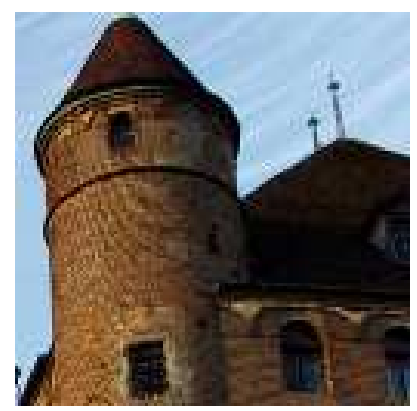

(a)

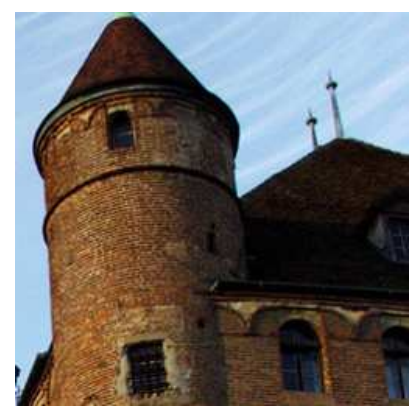

(b)
Fig. 4. Simulation result using four images undersampled by two (a). The original double resolution image is perfectly reconstructed (b).

\section{DISCUSSION}

Using the decomposition presented in Section 4, the projection of an $M N$-dimensional sample vector onto a $2 L+$ 1-dimensional space is reduced to a projection of an $M$ dimensional vector onto an $S$-dimensional space, with $S$ the number of overlapping Fourier coefficients. This also allows us to eliminate the components that do not contribute to the minimization function. If the signals are undersampled by less than two, the solution can be computed analytically. For signals that are undersampled by more than two, the offsets can be found by evaluating the projection on a regular grid of possible offset values. In this way, a first approximation of the global minimum is obtained. It can then be refined using a standard minimization algorithm like gradient descent.

In this paper, the images are described as a linear combination of Fourier basis vectors. However, this method can also be generalized to other bases, such as piecewise polynomials, gaussians, etc. As long as the continuous-time signal can be written as a linear combination of known basis vectors, and multiple sets of samples with unknown offsets are available, our method can be applied to reconstruct the original signal.

Next to super-resolution imaging, this algorithm can also be applied for demosaicing from multiple images. Whereas a standard algorithm based on only one image can contain color aliasing artifacts, they can be completely removed by taking multiple images.

The above simulation results were obtained using an ideal, noise-free setup. In future work, we plan to analyze the sensitivity of our method to noise and test it on a practical setup.

\section{CONCLUSIONS}

We presented a new algorithm to estimate unknown offsets between multiple arbitrarily aliased sets of samples and reconstruct the original signal. The offsets are computed using a subspace projection method. The projection is decomposed into different independent projections on spaces with smaller sizes, reducing the computational complexity. Once the offsets are known, the original signal is reconstructed as the solution of a set of linear equations in the unknown basis components. These basis components can be Fourier coefficients, or the coefficients in any other known basis. Our algorithm is applied to super-resolution imaging in a simulation, showing good results.

\section{REFERENCES}

[1] Pina Marziliano and Martin Vetterli, "Reconstruction of irregularly sampled discrete-time bandlimited signals with unknown sampling locations," IEEE Transactions on Signal Processing, vol. 48, no. 12, pp. 3462-3471, December 2000.

[2] Patrick Vandewalle, Luciano Sbaiz, Joos Vandewalle, and Martin Vetterli, "How to take advantage of aliasing in bandlimited signals," in IEEE Conference on Acoustics, Speech and Signal Processing, May 2004, vol. 3, pp. 948-951.

[3] R. Y. Tsai and T. S. Huang, "Multiframe image restoration and registration," in Advances in Computer Vision and Image Processing, vol. 1, pp. 317-339. JAI Press, 1984.

[4] Sean Borman and Robert Stevenson, "Spatial resolution enhancement of low-resolution image sequences a comprehensive review with directions for future research," Tech. Rep., University of Notre Dame, 1998.

[5] Sung Cheol Park, Min Kyu Park, and Moon Gi Kang, "Super-resolution image reconstruction: A technical overview," IEEE Signal Processing Magazine, vol. 20, no. 3, pp. 21-36, May 2003. 